\title{
Orbifold instantons, moment maps, and Yang-Mills theory with sources
}

\author{
Tatiana A. Ivanova, ${ }^{1, *}$ Olaf Lechtenfeld, ${ }^{2, \dagger}$ Alexander D. Popov, ${ }^{2, \ddagger}$ and Richard J. Szabo ${ }^{3, \S}$ \\ ${ }^{1}$ Bogoliubov Laboratory of Theoretical Physics, JINR, 141980 Dubna, Moscow Region, Russia \\ ${ }^{2}$ Institut für Theoretische Physik and Riemann Center for Geometry and Physics, Leibniz Universität Hannover, \\ Appelstraße 2, 30167 Hannover, Germany \\ ${ }^{3}$ Department of Mathematics, Heriot-Watt University, Colin Maclaurin Building, Riccarton, Edinburgh EH14 4AS, United Kingdom; \\ Maxwell Institute for Mathematical Sciences, Edinburgh, United Kingdom; and The Tait Institute, Edinburgh, United Kingdom
}

(Received 20 October 2013; published 26 November 2013)

\begin{abstract}
We revisit the problem of constructing instantons on ADE orbifolds $\mathbb{R}^{4} / \Gamma$ and point out some subtle relations with the complex structure on the orbifold. We consider generalized instanton equations on $\mathbb{R}^{4} / \Gamma$ which are BPS equations for the Yang-Mills equations with an external current. The relation between level sets of the moment maps in the hyper-Kähler quotient construction of the instanton moduli space and sources in the Yang-Mills equations is discussed. We describe two types of spherically symmetric $\Gamma$-equivariant connections on complex $\mathrm{V}$ bundles over $\mathbb{R}^{4} / \Gamma$ which are tailored to the way in which the orbifold group acts on the fibers. Some explicit Abelian and non-Abelian SU(2)-invariant solutions to the instanton equations on the orbifold are worked out.
\end{abstract}

DOI: $10.1103 /$ PhysRevD.88.105026

PACS numbers: 11.15.-q, 11.27.+d

\section{INTRODUCTION AND SUMMARY}

Instantons in Yang-Mills theory [1] and gravity [2,3] play an important role in modern field theory [4-6]. They are nonperturbative configurations which solve first-order (anti-)self-duality equations for the gauge field and the Riemann curvature tensor, respectively. The construction of gauge instantons can be described systematically in the framework of twistor theory $[7,8]$ and by the ADHM construction [9]. There are also many methods for constructing gravitational instantons including twistor theory [8] and the hyper-Kähler quotient construction [10] based on the hyper-Kähler moment map introduced in Ref. [11].

In this paper, we revisit the problem of constructing instantons on the $\mathrm{ADE}$ orbifolds $\mathbb{R}^{4} / \Gamma$. The corresponding instanton moduli spaces are of special interest in type-II string theory, where they can be realized as Higgs branches of certain quiver gauge theories which appear as worldvolume field theories on $\mathrm{D} p$ branes in a $\mathrm{D} p-\mathrm{D}(p+4)$ system with the $\mathrm{D}(p+4)$ branes located at the fixed point of the orbifold [12]. The ADHM equations can be identified with the vacuum equations of the supersymmetric gauge theory, and the structure of the vacuum moduli space provides an important example of resolution of spacetime singularities by stringy effects in the form of D-brane probes. We point out in particular some salient relations between the construction of instantons and complex structures on $\mathbb{R}^{4} / \Gamma$.

Kronheimer [10] considers $\Gamma$-equivariant solutions of the matrix equations:

\footnotetext{
*ita@theor.jinr.ru

†lechtenf@itp.uni-hannover.de

popov@itp.uni-hannover.de

§R.J.Szabo@hw.ac.uk
}

$$
\begin{aligned}
& {\left[W_{2}, W_{3}\right]+\left[W_{1}, W_{4}\right]=\Xi_{1},} \\
& {\left[W_{3}, W_{1}\right]+\left[W_{2}, W_{4}\right]=\Xi_{2},} \\
& {\left[W_{1}, W_{2}\right]+\left[W_{3}, W_{4}\right]=\Xi_{3},}
\end{aligned}
$$

where $\Gamma$ is a finite subgroup of the Lie group SU(2) acting on the fundamental representation $\mathbb{C}^{2} \cong \mathbb{R}^{4} ; W_{\mu}$, with $\mu=$ $1,2,3,4$, are matrices taking values in, e.g. ${ }^{1}$ the Lie algebra $\mathfrak{t}(N)$; and $\Xi_{a}$, with $a=1,2,3$, are matrices in the center $\mathfrak{h}$ of a subalgebra $g$ of $\mathfrak{u}(N)$. For $\Xi_{a}=0$, Eqs. (1.1) give the anti-self-dual Yang-Mills equations on the orbifold $\mathbb{C}^{2} / \Gamma$ reduced by translations. Their solutions satisfy the full Yang-Mills equations. In the general case, the equations (1.1) are interpreted as hyper-Kähler moment map quotient equations, and Hitchin shows [13] that one can similarly interpret the Bogomolny monopole equations and vortex equations. Kronheimer shows that the moduli space of solutions to Eqs. (1.1) in the Coulomb branch is a hyperKähler ALE space $M_{\xi}$, which is the minimal resolution

$$
M_{\xi} \rightarrow M_{0}
$$

of the orbifold $M_{0}=\mathbb{C}^{2} / \Gamma$. Here $\xi$ are parameters in the matrices $\Xi_{a}$ of Eqs. (1.1). Similar results were obtained in Refs. $[14,15]$ for SU(2)-invariant Yang-Mills instantons on $\mathbb{R}^{4}$ (see also Ref. [16]). Moreover, it was shown by Kronheimer and Nakajima [17] that there exists a bundle $\mathcal{E} \rightarrow M_{\xi}$ with Chern classes $c_{1}(\mathcal{E})=0$ and $c_{2}(\mathcal{E})=(\# \Gamma-1) /$ $\# \Gamma$ such that the moduli space of framed instantons on $\mathcal{E}$ satisfying the anti-self-dual Yang-Mills equations coincides with the base manifold $M_{\xi}$ itself. In the limit $\xi=0$, one obtains $\mathbb{C}^{2} / \Gamma$ as the moduli space of minimal

\footnotetext{
${ }^{1}$ In type-II string theory in the presence of orientifold $\mathrm{O}(p+4)$ planes, one should use instead the Lie algebras of orthogonal or symplectic Lie groups.
} 
fractional instantons on the $\mathrm{V}$ bundle $\mathcal{E}$ over the orbifold $M_{0}=\mathbb{C}^{2} / \Gamma$.

In this paper, we consider gauge instanton equations with matrices $\Xi_{a}$ on the orbifold $\mathbb{C}^{2} / \Gamma \cong \mathbb{R}^{4} / \Gamma$ and show that the choices of $\Xi_{a} \neq 0$ correspond to sources in the Yang-Mills equations. For gauge potentials on $\mathbb{R}^{4} / \Gamma$ with $\Gamma=\mathbb{Z}_{k+1}$, we analyze solutions of $\Gamma$ equivariance conditions in two different $\mathrm{SU}(2)$-invariant bases adapted to the spherical symmetry. Recall that one can write a realization of the Lie algebra $\mathfrak{S} \mathfrak{D}(4) \cong \mathfrak{S} \mathfrak{H}(2) \oplus \mathfrak{S} \mathfrak{H}(2)$ in terms of vector fields on $\mathbb{R}^{4} / \Gamma$ as

$$
E^{a}=-\eta_{\mu \nu}^{a} y^{\mu} \frac{\partial}{\partial y^{\nu}} \quad \text { and } \quad \tilde{E}^{a}=-\bar{\eta}_{\mu \nu}^{a} y^{\mu} \frac{\partial}{\partial y^{\nu}},
$$

where $\eta_{\mu \nu}^{a}$ and $\bar{\eta}_{\mu \nu}^{a}$ are components of the self-dual and anti-self-dual 't Hooft tensors [18], and $y^{\mu}$ are local coordinates on $\mathbb{R}^{4} / \Gamma$. The commutation relations between these vector fields are

$$
\begin{aligned}
& {\left[E^{a}, E^{b}\right]=2 \varepsilon^{a b c} E^{c},} \\
& {\left[\tilde{E}^{a}, \tilde{E}^{b}\right]=2 \varepsilon^{a b c} \tilde{E}^{c}, \quad \text { and }} \\
& {\left[E^{a}, \tilde{E}^{b}\right]=0 .}
\end{aligned}
$$

Introducing complex coordinates $z^{1}=y^{1}+\mathrm{i} y^{2}$ and $z^{2}=$ $y^{3}+\mathrm{i} y^{4}$ on $\mathbb{R}^{4} / \Gamma \cong \mathbb{C}^{2} / \Gamma$, one finds that the vector fields $\tilde{E}^{a}$ preserve this complex structure, but the vector fields $E^{a}$ do not; i.e., the group $\mathrm{SU}(2)$ acting on $\mathbb{C}^{2}$ is generated by $\left(\tilde{E}^{a}\right)$. Furthermore, the actions of the corresponding Lie derivatives are given by

$$
\mathcal{L}_{\tilde{E}^{b}} e^{a}=0 \quad \text { and } \quad \mathcal{L}_{\tilde{E}^{b}} \tilde{e}^{a}=2 \varepsilon_{b c}^{a} \tilde{e}^{c},
$$

where $e^{a}=e_{\mu}^{a} \mathrm{~d} y^{\mu}$ and $\tilde{e}^{a}=\tilde{e}_{\mu}^{a} \mathrm{~d} y^{\mu}$ are one-forms dual to the vector fields $E^{a}$ and $\tilde{E}^{a}$, respectively. We show that both bases of the one-forms $\left(e^{a}, \mathrm{~d} r\right)$ and $\left(\tilde{e}^{a}, \mathrm{~d} r\right)$, with $r^{2}=$ $\delta_{\mu \nu} y^{\mu} y^{\nu}$, can be used for describing spherically symmetric instanton configurations, but due to Eq. (1.5), the basis $\left(e^{a}, \mathrm{~d} r\right)$ is more suitable for connections on V bundles $\mathcal{E}$ with trivial action of the finite group $\Gamma \subset \mathrm{SU}(2)$, while the basis $\left(\tilde{e}^{a}, \mathrm{~d} r\right)$ is more suitable for connections on $\mathrm{V}$ bundles $\mathcal{E}$ with nontrivial $\Gamma$ action on the fibers of $\mathcal{E}$. Explicit examples of Abelian and non-Abelian SU(2)-invariant instanton solutions on $\mathbb{R}^{4} / \mathbb{Z}_{k+1}$ are worked out below.

The structure of the remainder of this paper is as follows: In Sec. II, we consider generalized instanton equations on $\mathbb{R}^{4}$ which reduce to Eq. (1.1) and show that they correspond to BPS-type equations for Yang-Mills theory with sources. In Sec. III, we extend these equations to the ADE quotient singularities $\mathbb{R}^{4} / \Gamma$, focusing on the special case $\Gamma=\mathbb{Z}_{k+1}$. In Sec. IV, we study the moduli spaces of translationally invariant instantons on $\mathbb{R}^{4} / \Gamma$ via the hyper-Kähler quotient construction. In Sec. V, we consider the construction of spherically symmetric instanton solutions on $\mathbb{R}^{4} / \Gamma$ and make some preliminary comments concerning the structure of the instanton moduli spaces, though a detailed description of these moduli spaces is beyond the scope of the present work.

\section{INSTANTON EQUATIONS ON $\mathbb{R}^{4}$}

\section{A. Euclidean space $\mathbb{R}^{4}$}

Consider the two-forms

$$
\omega^{a}:=\frac{1}{2} \eta_{\mu \nu}^{a} \mathrm{~d} y^{\mu} \wedge \mathrm{d} y^{\nu},
$$

where $y^{\mu}$ are coordinates on $\mathbb{R}^{4}$ and $\omega_{\mu \nu}^{a}:=\eta_{\mu \nu}^{a}$ are components of the 't Hooft tensors given by the formulas

$$
\eta_{b c}^{a}=\varepsilon_{b c}^{a} \quad \text { and } \quad \eta_{b 4}^{a}=-\eta_{4 b}^{a}=\delta_{b}^{a} .
$$

Here $\varepsilon_{23}^{1}=1, \mu, \nu, \ldots=1,2,3,4$, and $a, b, \ldots=1,2,3$. The forms $\omega^{a}$ are symplectic and self-dual,

$$
\mathrm{d} \omega^{a}=0 \text { and } * \omega^{a}=\omega^{a},
$$

where $*$ is the Hodge duality operator for the flat metric

$$
g=\delta_{\mu \nu} \mathrm{d} y^{\mu} \otimes \mathrm{d} y^{\nu}
$$

on $\mathbb{R}^{4}$.

Using the metric (2.4), we introduce three complex structures $J^{a}=\omega^{a} \circ g^{-1}$ on $\mathbb{R}^{4}$ with components

$$
\left(J^{a}\right)_{\nu}^{\mu}=\omega_{\nu \lambda}^{a} \delta^{\lambda \mu},
$$

so that $\left(\mathbb{R}^{4}, J^{a}\right) \cong \mathbb{C}_{J^{a}}^{2}$. The space $\mathbb{R}^{4}$ is hyper-Kähler; i.e., it is Kähler with respect to each of the complex structures in Eq. (2.5). We choose one of them, $J^{3}:=J$, to identify $\mathbb{R}^{4}$ and $\mathbb{C}^{2} \cong\left(\mathbb{R}^{4}, J\right)$. With respect to $J$, the complex two-form

$$
\omega_{\mathbb{C}}=\omega^{1}+\mathrm{i} \omega^{2}
$$

is closed and holomorphic; i.e., $\omega_{\mathbb{C}}$ is a $(2,0)$-form.

\section{B. Instanton equations}

Let $\mathcal{E}$ be a rank- $N$ complex vector bundle over $\mathbb{R}^{4} \cong \mathbb{C}^{2}$. We endow this bundle with a connection $\mathcal{A}=\mathcal{A}_{\mu} \mathrm{d} y^{\mu}$ of curvature $\mathcal{F}=\mathrm{d} \mathcal{A}+\mathcal{A} \wedge \mathcal{A}=\frac{1}{2} \mathcal{F}_{\mu \nu} \mathrm{d} y^{\mu} \wedge \mathrm{d} y^{\nu}$ taking values in the Lie algebra $\mathfrak{t}(N)$. Let us constrain the curvature $\mathcal{F}$ by the equations

$$
* \mathcal{F}+\mathcal{F}=2 \omega^{a} \Xi_{a},
$$

where the functions $\Xi_{a}$ belong to $\mathfrak{t}(N)$. Solutions to this equation of finite topological charge are called (generalized) instantons. If $\Xi_{a}$ belong to the center $\mathfrak{u}(1)$ of $\mathfrak{t}(N)$ and $\mathrm{d} \Xi_{a}=0$, then solutions to the equations (2.7) satisfy the Yang-Mills equations on $\mathbb{R}^{4}$. If $\Xi_{a}$ do not belong to this center, $^{2}$ then Eqs. (2.7) are BPS-type equations for Yang-Mills theory with sources which vanish only if $\Xi_{a}$ are constant and $\Xi_{a} \in \mathfrak{u}(1) \subset \mathfrak{u}(N)$ for $a=1,2,3$. Indeed, from Eqs. (2.7), we get

\footnotetext{
${ }^{2}$ Later on, we will consider an important example of such noncentral elements $\Xi_{a}$.
} 


$$
\begin{array}{r}
\mathrm{d} * \mathcal{F}+\mathcal{A} \wedge * \mathcal{F}-* \mathcal{F} \wedge \mathcal{A} \\
\quad=2 \omega^{a} \wedge\left(\mathrm{d} \Xi_{a}+\left[\mathcal{A}, \Xi_{a}\right]\right),
\end{array}
$$

which after taking the Hodge dual can be rewritten as

$\partial_{\mu} \mathcal{F}_{\mu \nu}+\left[\mathcal{A}_{\mu}, \mathcal{F}_{\mu \nu}\right]=4 \omega_{\mu \nu}^{a}\left(\partial_{\mu} \Xi_{a}+\left[\mathcal{A}_{\mu}, \Xi_{a}\right]\right)$.

The current

$j_{\mu}:=4 \omega_{\nu \mu}^{a} D_{\nu} \Xi_{a} \quad$ with $\quad D_{\mu} \Xi_{a}:=\partial_{\mu} \Xi_{a}+\left[\mathcal{A}_{\mu}, \Xi_{a}\right]$

satisfies the covariant continuity equation

$$
D_{\mu} j_{\mu}=0 \text {, }
$$

as required for minimal coupling of an external current in the Yang-Mills equations.

\section{Variational equations}

To formulate the generalized instanton equations (2.7) as absolute minima of Euler-Lagrange equations derived from an action principle, we note that the presence of the current [Eq. (2.10)] in the Yang-Mills equations (2.9) requires the addition of the term

$$
\frac{1}{2} \operatorname{tr} j_{\mu} \mathcal{A}_{\mu}
$$

in the standard Yang-Mills Lagrangian

$$
\mathcal{L}_{\mathrm{YM}}=-\frac{1}{8} \operatorname{tr} \mathcal{F}_{\mu \nu} \mathcal{F}_{\mu \nu} .
$$

Up to a total derivative, the term in Eq. (2.12) is equivalent to the term

$$
\frac{1}{2} \omega_{\mu \nu}^{a} \operatorname{tr} \mathcal{F}_{\mu \nu} \Xi_{a} .
$$

After adding the term in Eq. (2.14), together with the nondynamical term

$$
-3 \operatorname{tr} \Xi_{a} \Xi_{a}
$$

and the topological density

$$
-\frac{1}{2} \varepsilon_{\mu \nu \lambda \sigma} \operatorname{tr} \mathcal{F}_{\mu \nu} \mathcal{F}_{\lambda \sigma},
$$

we obtain the Lagrangian

$$
\mathcal{L}=-\frac{1}{4} \operatorname{tr}\left(\mathcal{F}_{\mu \nu}^{+}-\omega_{\mu \nu}^{a} \Xi_{a}\right)\left(\mathcal{F}_{\mu \nu}^{+}-\omega_{\mu \nu}^{a} \Xi_{a}\right),
$$

where

$$
\mathcal{F}^{+}=\frac{1}{2}(* \mathcal{F}+\mathcal{F})
$$

is the self-dual part of the curvature two-form $\mathcal{F}$. In the following, we will consider constant matrices $\Xi_{a}$ for which
(2.15) becomes constant and the term in Eq. (2.14) is topological. Constant matrices of the form $\Xi_{a}=\mathrm{i} \xi_{a} \mathbf{1}_{N}$ correspond to D3 branes in a nonzero $B$ field in string theory and can be described in terms of a noncommutative deformation of Yang-Mills theory on the space $\mathbb{R}^{4}$ (see e.g., Refs. [19,20]).

\section{INSTANTON EQUATIONS ON $\mathbb{R}^{4} / \Gamma$}

\section{A. Orbifold $\mathbb{R}^{4} / \Gamma$}

The complex structure $J=J^{3}$, introduced in Eq. (2.5), defines the complex coordinates

$$
z^{1}=y^{1}+\mathrm{i} y^{2} \quad \text { and } \quad z^{2}=y^{3}+\mathrm{i} y^{4}
$$

on $\mathbb{R}^{4} \cong \mathbb{C}^{2}$, where $y^{\mu}$ are real coordinates. The Lie group SU(2) naturally acts on the vector space $\mathbb{C}^{2}$ with the coordinates in Eq. (3.1). We are interested in the Kleinian orbifolds $\mathbb{C}^{2} / \Gamma$, where $\Gamma$ is a finite subgroup of $\operatorname{SU}(2)$. They have an ADE classification in which $\Gamma$ is associated with the extended Dynkin diagram of a simply laced simple Lie algebra. For the $A_{k}$-type simple singularities, corresponding to the cyclic group $\Gamma=\mathbb{Z}_{k+1}$ of order $k+1$, explicit descriptions of instantons will be readily available. However, most of our results can be generalized to the other ADE groups $\Gamma$ corresponding to non-Abelian orbifolds $\mathbb{C}^{2} / \Gamma$.

The action of $\Gamma=\mathbb{Z}_{k+1}$ on $\mathbb{C}^{2}$ is given by

$$
\left(z^{1}, z^{2}\right) \mapsto\left(\zeta z^{1}, \zeta^{-1} z^{2}\right),
$$

where

$$
\zeta=\exp \left(\frac{2 \pi \mathrm{i}}{k+1}\right) \quad \text { with } \quad \zeta^{k+1}=1
$$

is a primitive $(k+1)$-th root of unity. This action has a single isolated fixed point at the origin $\left(z^{1}, z^{2}\right)=(0,0)$. The orbifold $\mathbb{C}^{2} / \Gamma$ is defined as the set of equivalence classes on $\mathbb{C}^{2}$ with respect to the equivalence relation

$$
\left(\zeta z^{1}, \zeta^{-1} z^{2}\right) \equiv\left(z^{1}, z^{2}\right)
$$

and it has a singularity at the origin. The metric on $\mathbb{C}^{2} / \Gamma$ is

$$
g=\mathrm{d} z^{1} \otimes \mathrm{d} \bar{z}^{\overline{1}}+\mathrm{d} z^{2} \otimes \mathrm{d} \bar{z}^{2},
$$

where the coordinates $\bar{z}^{\overline{1}}, \bar{z}^{\overline{2}}$ are complex-conjugated to $z^{1}, z^{2}$.

\section{B. $V$ bundles on $\mathbb{C}^{2} / \Gamma$}

A V bundle on $\mathbb{C}^{2} / \Gamma$ is a $\Gamma$-equivariant bundle over $\mathbb{C}^{2}$; i.e., a vector bundle on $\mathbb{C}^{2}$ with a $\Gamma$ action on the fibers which is compatible with the action of $\Gamma$ on $\mathbb{C}^{2}$. The orbifold group $\Gamma=\mathbb{Z}_{k+1}$ has $k+1$ one-dimensional irreducible representations such that the generator of $\mathbb{Z}_{k+1}$ acts on the $\ell$ th $\Gamma$ module as multiplication by $\zeta^{\ell}$ for $\ell=0,1, \ldots, k$. Let us denote by $\mathcal{E}_{\ell}$ complex $\mathrm{V}$ bundles over $\mathbb{C}^{2} / \Gamma$ of rank $N_{\ell}$ on which $\Gamma$ acts in the $\ell$ th irreducible representation as 


$$
\boldsymbol{v}_{\ell} \mapsto \zeta^{\ell} \boldsymbol{v}_{\ell} \text { for } \boldsymbol{v}_{\ell} \in \mathbb{C}^{N_{\ell}}
$$

on a generic fiber $\mathbb{C}^{N_{\ell}}$ of $\mathcal{E}_{\ell}$. Then any complex V bundle $\mathcal{E}$ over $\mathbb{C}^{2} / \Gamma$ of rank $N$ can be decomposed into isotopical components as a Whitney sum

$$
\mathcal{E}=\bigoplus_{\ell=0}^{k} \mathcal{E}_{\ell}
$$

and its structure group is of the form

$$
\prod_{\ell=0}^{k} \mathrm{U}\left(N_{\ell}\right) \quad \text { with } \quad \sum_{\ell=0}^{k} N_{\ell}=N .
$$

From Eq. (3.6), it follows that the action of the point group on the $\mathrm{V}$ bundle [Eq. (3.7)] is given by the unitary matrices

$$
v \mapsto \gamma_{\Gamma}(v) \quad \text { with } \quad \gamma_{\Gamma}=\bigoplus_{\ell=0}^{k} \zeta^{\ell} \mathbf{1}_{N_{\ell}}
$$

on vectors $v=\left(v_{\ell}\right)_{\ell=0}^{k}$ in the generic fiber $\mathbb{C}^{N}=$ $\bigoplus_{\ell=0}^{k} \mathbb{C}^{N_{\ell}}$ of $\mathcal{E}$.

Simplifying the situation discussed in the previous section, we choose matrices $\Xi_{a}$ in the form

$$
\Xi_{a}=\bigoplus_{\ell=0}^{k} \mathrm{i} \xi_{a}^{\ell} \mathbf{1}_{N_{\ell}}
$$

where $\xi_{a}^{\ell} \in \mathbb{R}$ are constants. The matrices in Eq. (3.10) belong to the center of the Lie algebra of the gauge group [Eq. (3.8)]. The diagonal $U(1)$ subgroup of scalars in Eq. (3.8) acts trivially on $(\mathcal{E}, \mathcal{A})$, so we can factor the gauge group in Eq. (3.8) by this U(1) subgroup to get the quotient group

$$
G:=\left(\prod_{\ell=0}^{k} \mathrm{U}\left(N_{\ell}\right)\right) / \mathrm{U}(1) .
$$

Then, the Lie algebra $g$ of $G$ is the traceless part of the Lie algebra of Eq. (3.8), and one should impose on $\xi_{a}^{\ell}$ in Eq. (3.10) the tracelessness condition

$$
\sum_{\ell=0}^{k} \xi_{a}^{\ell} N_{\ell}=0
$$

which defines the center $\mathfrak{h}$ of $\mathfrak{g}$.

\section{C. $\Gamma$-equivariant connections}

Consider a one-form

$W=W_{\mu} \mathrm{d} y^{\mu}=W_{z^{1}} \mathrm{~d} z^{1}+W_{z^{2}} \mathrm{~d} z^{2}+W_{\bar{z}^{1}} \mathrm{~d} \bar{z}^{\overline{1}}+W_{\bar{z}^{\bar{z}}} \mathrm{~d} \bar{z}^{\overline{2}}$

on $\mathbb{R}^{4} \cong \mathbb{C}^{2}$ which is invariant under the action of $\Gamma \subset \mathrm{SU}(2) \subset \mathrm{SO}(4)$ defined by Eq. (3.2). Then, on the components
$W_{z^{1}}=\frac{1}{2}\left(W_{1}-\mathrm{i} W_{2}\right) \quad$ and $\quad W_{z^{2}}=\frac{1}{2}\left(W_{3}-\mathrm{i} W_{4}\right)$,

the action of $\Gamma$ is given by

$$
W_{z^{1}} \mapsto \zeta^{-1} W_{z^{1}} \quad \text { and } \quad W_{z^{2}} \mapsto \zeta W_{z^{2}}
$$

The action of $\Gamma$ on the components $\mathcal{A}_{\mu}$ of any unitary connection $\mathcal{A}=\mathcal{A}_{\mu} \mathrm{d} y^{\mu}$ on a Hermitian $\mathrm{V}$ bundle [Eq. (3.7)] is given by a combination of the spacetime action [Eq. (3.15)] and the adjoint action generated by matrices from Eq. (3.9) as

$$
\mathcal{A}_{z^{1}} \mapsto \zeta^{-1} \gamma_{\Gamma} \mathcal{A}_{z^{1}} \gamma_{\Gamma}^{-1} \quad \text { and } \quad \mathcal{A}_{z^{2}} \mapsto \zeta \gamma_{\Gamma} \mathcal{A}_{z^{2}} \gamma_{\Gamma}^{-1}
$$

The corresponding $\Gamma$-equivariance conditions require that the connection defines a covariant representation of the orbifold group, in the sense that

$\gamma_{\Gamma} \mathcal{A}_{z^{1}} \gamma_{\Gamma}^{-1}=\zeta \mathcal{A}_{z^{1}} \quad$ and $\quad \gamma_{\Gamma} \mathcal{A}_{z^{2}} \gamma_{\Gamma}^{-1}=\zeta^{-1} \mathcal{A}_{z^{2}}$.

It is easy to see that the solutions to the constraint equations (3.17) are given by block off-diagonal matrices

$$
\begin{aligned}
\mathcal{A}_{z^{1}} & =\left(\begin{array}{ccccc}
0 & 0 & \cdots & 0 & \psi_{k+1} \\
\psi_{1} & 0 & \ddots & 0 & 0 \\
0 & \psi_{2} & \ddots & \vdots & 0 \\
\vdots & \ddots & \ddots & 0 & 0 \\
0 & \cdots & 0 & \psi_{k} & 0
\end{array}\right) \text { and } \\
\mathcal{A}_{z^{2}} & =\left(\begin{array}{ccccc}
0 & \phi_{1} & 0 & \cdots & 0 \\
0 & 0 & \phi_{2} & \ddots & \vdots \\
\vdots & \vdots & \ddots & \ddots & 0 \\
0 & 0 & \cdots & 0 & \phi_{k} \\
\phi_{k+1} & 0 & \cdots & 0 & 0
\end{array}\right)
\end{aligned}
$$

together with $\mathcal{A}_{\bar{z}^{1}}=-\mathcal{A}_{z^{1}}^{\dagger}$ and $\mathcal{A}_{\bar{z}^{\overline{2}}}=-\mathcal{A}_{z^{2}}^{\dagger}$. Here the bundle morphisms $\psi_{\ell+1}: \mathcal{E}_{\ell} \rightarrow \mathcal{E}_{\ell+1}$ and $\phi_{\ell+1}: \mathcal{E}_{\ell+1} \rightarrow \mathcal{E}_{\ell}$ are bifundamental scalar fields given fiberwise by matrices

$$
\begin{aligned}
& \psi_{\ell+1} \in \operatorname{Hom}\left(\mathbb{C}^{N_{\ell}}, \mathbb{C}^{N_{\ell+1}}\right) \quad \text { and } \\
& \phi_{\ell+1} \in \operatorname{Hom}\left(\mathbb{C}^{N_{\ell+1}}, \mathbb{C}^{N_{\ell}}\right)
\end{aligned}
$$

for $\ell=0,1, \ldots, k$ (with indices read modulo $k+1$ ). Substitution of Eq. (3.18) into Eq. (2.7) then yields the generalized instanton equations on the orbifold $\mathbb{C}^{2} / \Gamma$. The transformations in Eq. (3.15) are defined for the holonomic basis $\mathrm{d} y^{\mu}$ of one-forms on $\mathbb{R}^{4} / \Gamma$ and can differ for other bases of one-forms, leading to modifications of the formulas in Eqs. (3.16), (3.17), and (3.18). 


\section{TRANSLATIONALLY INVARIANT INSTANTONS}

\section{A. Matrix equations}

Consider translationally invariant connections $\mathcal{A}$ on the $\mathrm{V}$ bundle in Eq. (3.7) over $\mathbb{C}^{2} / \Gamma$ satisfying the equations (2.7) with $\Xi_{a}$ given in Eq. (3.10); i.e., we assume that $\mathcal{A}_{\mu}$ are independent of the coordinates $y^{\mu}$, which reduces Eq. (2.7) to the matrix equations (1.1) with $W_{\mu}:=\mathcal{A}_{\mu}$. Denoting $B_{1}:=\mathcal{A}_{z^{1}}$ and $B_{2}:=\mathcal{A}_{z^{2}}$ for $\mathcal{A}$ given by Eq. (3.18) with constant matrices $\psi_{\ell+1}$ and $\phi_{\ell+1}$ for $\ell=0,1, \ldots, k$, we obtain the equations

$$
\frac{1}{2} \eta_{\mu \nu}^{a}\left[W_{\mu}, W_{\nu}\right]=\Xi_{a}
$$

which can be rewritten as

$$
\begin{gathered}
{\left[B_{1}, B_{2}\right]=-\frac{\mathrm{i}}{4}\left(\Xi_{1}-\mathrm{i} \Xi_{2}\right)=: \Xi_{\mathbb{C}},} \\
{\left[B_{1}, B_{1}^{\dagger}\right]+\left[B_{2}, B_{2}^{\dagger}\right]=-\frac{\mathrm{i}}{2} \Xi_{3}=: \Xi_{\mathbb{R}} .}
\end{gathered}
$$

Solutions to these equations satisfy the reduced Yang-Mills equations (2.9) with the external source

$$
j_{\mu}=-4 \eta_{\mu \nu}^{a}\left[W_{\nu}, \Xi_{a}\right],
$$

where $W_{\mu}$ is given by Eq. (3.18) and $\Xi_{a}$ by Eq. (3.10).

\section{B. Hyper-Kähler quotients}

The reduced equations (4.1) [and also the instanton equations (2.7)] can be interpreted as hyper-Kähler moment map equations. For this, recall that if $\left(M, g, \omega^{a}\right)$ is a hyper-Kähler manifold with an action of a Lie group $G$ which preserves the metric $g$ and the three Kähler forms ${ }^{3}$ $\omega^{a}$, then one can define three moment maps

$$
\mu^{a}: M \rightarrow \mathrm{g}^{*}
$$

taking values in the dual $\mathrm{g}^{*}$ of the Lie algebra $\mathrm{g}$ of $G$ such that, for each $\xi \in \mathfrak{g}$ with a triholomorphic Killing vector field $L_{\xi}$ generated by the $G$ action on $M$, the functions in Eq. (4.5) satisfy the equations

$$
\left.\left\langle\mathrm{d} \mu^{a}, \xi\right\rangle=L_{\xi}\right\lrcorner \omega^{a},
$$

where $\langle-,-\rangle$ is the dual pairing between elements of $\mathrm{g}^{*}$ and $\mathfrak{g}$, and $\lrcorner$ denotes the contraction of vector fields and differential forms. Denoting by $\mu=\left(\mu^{1}, \mu^{2}, \mu^{3}\right)$ the vector-valued moment map

$$
\mu: M \rightarrow \mathbb{R}^{3} \otimes \mathfrak{g}^{*},
$$

\footnotetext{
${ }^{3}$ They are Kähler with respect to the three complex structures $J^{a}=\omega^{a} \circ g^{-1}$. With respect to the complex structure $J^{3}$, the two-form $\omega_{\mathbb{R}}=\omega^{3}$ is Kähler, and $\omega_{\mathbb{C}}=\omega^{1}+\mathrm{i} \omega^{2}$ is holomorphic.
}

we can consider the $G$-invariant level set

$$
\mu^{-1}(\Xi)
$$

which defines a submanifold of the manifold $M$, where $\Xi=\left(\Xi_{1}, \Xi_{2}, \Xi_{3}\right) \in \mathbb{R}^{3} \otimes \mathfrak{h}^{*}$ and $\mathfrak{h}$ is the center of $\mathfrak{g}$. Then one can define the hyper-Kähler quotient as (see e.g., Refs. $[10,11,13])$

$$
M_{\xi}=\mu^{-1}(\Xi) / / / G,
$$

where $\xi=\left(\xi_{a}^{\ell}\right)$ are parameters defining $\Xi=\left(\Xi_{a}\right) \in$ $\mathbb{R}^{3} \otimes \mathfrak{h}^{*}$. The hyper-Kähler metric on $M$ descends to a hyper-Kähler metric on the quotient $M_{\xi}$. When the group action is free, the reduced space $M_{\xi}$ is a hyper-Kähler manifold of dimension $\operatorname{dim} M_{\xi}=\operatorname{dim} M-4 \operatorname{dim} G$.

In the case of the matrix model in Eq. (4.1), the manifold $M$ is the flat hyper-Kähler manifold

$$
M=\mathbb{R}^{4} \otimes \mathfrak{u}(N),
$$

the group $G$ is given in Eq. (3.11) and the three moment maps are ${ }^{4}$

$$
\mu^{a}(W)=\frac{1}{2} \eta_{\mu \nu}^{a}\left[W_{\mu}, W_{\nu}\right] \in \mathfrak{t}(N) .
$$

Solutions of Eqs. (4.2) and (4.3) form a submanifold $\mu^{-1}(\Xi)$ of the manifold in Eq. (4.10), and by factoring with the gauge group in Eq. (3.11) [which for generic parameters $\xi=\left(\xi_{a}^{\ell}\right)$ acts freely on the solutions], we obtain the moduli space [Eq. (4.9)]. This moduli space was studied by Kronheimer [10], who showed that for $\Gamma=\mathbb{Z}_{k+1}$ and the Coulomb branch $N_{0}=N_{1}=\cdots=$ $N_{k}=1$, it is a smooth, four-dimensional, asymptotically locally Euclidean (ALE) hyper-Kähler manifold $M_{\xi}$ with a metric defined by the parameters $\xi=\left(\xi_{a}^{\ell}\right)$. The ALE condition means that at asymptotic infinity of $M_{\xi}$, the metric approximates the Euclidean metric on the orbifold $\mathbb{C}^{2} / \Gamma$. Kronheimer also shows that $M_{\xi}$ is diffeomorphic to the minimal smooth resolution of the Kleinian singularity $M_{0}=\mathbb{C}^{2} / \Gamma$, regarded as the affine algebraic variety $x^{k+1}+y^{2}+z^{2}=0$ in $\mathbb{C}^{3}$. For the Hilbert-Chow map

$$
\pi: M_{\xi} \rightarrow M_{0},
$$

the exceptional divisor of the blowup is the set

$$
\pi^{-1}(0)=\bigcup_{\ell=0}^{k} \Sigma_{\ell}
$$

where $\Sigma_{\ell} \cong \mathbb{C} P^{1}$ and $k=\# \Gamma-1 .^{5}$ The parameters $\xi$ determine the periods of the three symplectic forms $\omega^{a}$ as

\footnotetext{
${ }^{4}$ We identify $\mathfrak{h t}(N)^{*}$ and $\mathfrak{t}(N)$.

${ }^{5}$ Recall that we consider $\Gamma=\mathbb{Z}_{k+1}$ for definiteness here, but many of these considerations generalize to the other Kleinian groups $\Gamma \subset \mathrm{SU}(2)$. In the general case, $N_{\ell}$ are the dimensions of the irreducible representations of the finite group $\Gamma$ in Kronheimer's construction.
} 


$$
\int_{\Sigma_{\ell}} \omega^{a}=\xi_{a}^{\ell}
$$

In the general case $N_{\ell} \geq 0$, one can also define a map $M_{\xi} \rightarrow M_{0}$ which is a resolution of singularities [21].

\section{Hermitian Yang-Mills connections}

The matrix $\Xi_{\mathbb{C}}$ in Eq. (4.2) parametrizes deformations of the complex structure on the $\mathrm{V}$ bundle $\mathcal{E}$, and it can be reabsorbed through a nonanalytic change of coordinates on the space in Eq. (4.10) [10,22]. Therefore, we may take $\Xi_{\mathbb{C}}=0$ without loss of generality; in this case, the ALE space $M_{\xi}$ is biholomorphic to the minimal resolution. In fact, the moduli spaces $M_{\xi}$ and $M_{\xi^{\prime}}$ are diffeomorphic for distinct $\xi$ and $\xi^{\prime}$ such that $\Xi_{\mathbb{R}} \neq 0$ for both sets of parameters. For $\Xi_{\mathbb{C}}=0$, we have $\Xi_{1}=\Xi_{2}=0$, and the equations (2.7) become the Hermitian Yang-Mills equations $[23,24]$

$$
* \mathcal{F}+\mathcal{F}=\omega^{3} \Xi_{3} .
$$

A connection $\mathcal{A}$ on $\mathcal{E}$ satisfying Eq. (4.15) is said to be a Hermitian Yang-Mills connection. It defines a holomorphic structure on $\mathcal{E}$, since from Eq. (4.15) it follows that the curvature $\mathcal{F}$ is of type $(1,1)$ with respect to the complex structure $J$, i.e.,

$$
\mathcal{F}^{2,0}=0=\mathcal{F}^{0,2}
$$

and the third equation from Eq. (4.15),

$$
\omega_{\mu \nu}^{3} \mathcal{F}_{\mu \nu}=\Xi_{3},
$$

means that, for $\Xi_{3}=\mathrm{i} \xi \mathbf{1}_{N}$, the $\mathrm{V}$ bundle $\mathcal{E}$ is (semi)stable $[23,24]$. In the special case $\Xi_{3}=0$, we get the standard anti-self-dual Yang-Mills equations

$$
* \mathcal{F}=-\mathcal{F} \text {. }
$$

\section{Translationally equivariant instantons}

Instead of constant matrices $\mathcal{A}_{\mu}$ which reduce Eq. (2.7) to the matrix equations (4.1), one can also consider the gauge potential

$$
\mathcal{A}=\frac{1}{2} \omega_{\mu \nu}^{a} \Xi_{a} y^{\mu} \mathrm{d} y^{\nu},
$$

where the commuting matrices $\Xi_{a}$ are given in Eq. (3.10). The connection in Eq. (4.19) is translationally invariant up to a gauge transformation and can be extended to the orbifold $T^{4} / \Gamma$, where $T^{4}$ is a four-dimensional torus. The curvature of $\mathcal{A}$ is

$$
\mathcal{F}=\mathrm{d} \mathcal{A}=\frac{1}{2} \omega_{\mu \nu}^{a} \Xi_{a} \mathrm{~d} y^{\mu} \wedge \mathrm{d} y^{\nu},
$$

providing in essence the three symplectic structures $\omega^{a}$ from Eq. (2.1).

\section{SPHERICALLY SYMMETRIC INSTANTONS}

\section{A. Cone $C\left(S^{3} / \Gamma\right)$}

The Euclidean space $\mathbb{R}^{4}$ can be regarded as a cone over the three-sphere $S^{3}$,

$$
\mathbb{R}^{4} \backslash\{0\}=C\left(S^{3}\right),
$$

with the metric

$$
g=\delta_{\mu \nu} \mathrm{d} y^{\mu} \otimes \mathrm{d} y^{\nu}=\mathrm{d} r^{2}+r^{2} \delta_{a b} e^{a} \otimes e^{b},
$$

where $r^{2}=\delta_{\mu \nu} y^{\mu} y^{\nu}$ and $\left(e^{a}\right)$ give a basis of left $\mathrm{SU}(2)$-invariant one-forms on $S^{3}$. One can define $e^{a}$ by the formula

$$
e^{a}:=-\frac{1}{r^{2}} \eta_{\mu \nu}^{a} y^{\mu} \mathrm{d} y^{\nu}
$$

where the 't Hooft tensors $\eta_{\mu \nu}^{a}$ are defined in Eq. (2.2). The one-forms $e^{a}$ are dual to the vector fields $E^{a}$ from Eq. (1.3). By using the identities

$\varepsilon_{b c}^{a} \eta_{\mu \nu}^{b} \eta_{\lambda \sigma}^{c}=\delta_{\mu \lambda} \eta_{\nu \sigma}^{a}-\delta_{\mu \sigma} \eta_{\nu \lambda}^{a}-\delta_{\nu \lambda} \eta_{\mu \sigma}^{a}+\delta_{\nu \sigma} \eta_{\mu \lambda}^{a}$,

$$
\delta_{a b} \eta_{\mu \nu}^{a} \eta_{\lambda \sigma}^{b}=\delta_{\mu \lambda} \delta_{\nu \sigma}-\delta_{\mu \sigma} \delta_{\nu \lambda}+\varepsilon_{\mu \nu \lambda \sigma},
$$

one can easily verify the Maurer-Cartan equations

$$
\mathrm{d} e^{a}+\varepsilon_{b c}^{a} e^{b} \wedge e^{c}=0
$$

and

$$
\omega^{a}=\frac{1}{2} \eta_{\mu \nu}^{a} \mathrm{~d} y^{\mu} \wedge \mathrm{d} y^{\nu}=\frac{1}{2} \eta_{\mu \nu}^{a} \hat{e}^{\mu} \wedge \hat{e}^{\nu}
$$

where

$$
\hat{e}^{a}:=r e^{a} \quad \text { and } \quad \hat{e}^{4}:=\mathrm{d} r .
$$

The relation (5.2) between the metric in Cartesian and spherical coordinates can be readily checked as well.

All formulas (5.2), (5.3), (5.4), (5.5), (5.6), (5.7), and (5.8) are also valid for the orbifold $\mathbb{C}^{2} / \Gamma$ after imposing the equivalence relation in Eq. (3.4), and the orbifold is a cone over the lens space $S^{3} / \Gamma$,

$$
\left(\mathbb{C}^{2} \backslash\{0\}\right) / \Gamma=C\left(S^{3} / \Gamma\right),
$$

with the metric (5.2). The one-forms [Eq. (5.3)] in the complex coordinates [Eq. (3.1)] have the form

$$
\begin{aligned}
& e^{1}+\mathrm{i} e^{2}=\frac{\mathrm{i}}{r^{2}}\left(z^{1} \mathrm{~d} z^{2}-z^{2} \mathrm{~d} z^{1}\right) \quad \text { and } \\
& e^{3}+\mathrm{i} e^{4}=\frac{\mathrm{i}}{r^{2}}\left(\bar{z}^{\overline{1}} \mathrm{~d} z^{1}+\bar{z}^{\overline{2}} \mathrm{~d} z^{2}\right)
\end{aligned}
$$

plus their complex-conjugated expressions. Hence, Eq. (5.10) defines two complex one-forms which are

\footnotetext{
${ }^{6}$ The orbifolds $S^{3} / \Gamma$ for arbitrary ADE point groups $\Gamma$ exhaust the possible Sasaki-Einstein manifolds in three dimensions.
} 
$(1,0)$-forms with respect to the complex structure $J=J^{3}$ defined in Eq. (2.5). The symplectic two-forms [Eq. (5.7)] and the complex structures [Eq. (2.5)] have the same components in the holonomic $\left(\mathrm{d} y^{\mu}, \frac{\partial}{\partial y^{\mu}}\right)$ and nonholonomic $\left(\hat{e}^{a}, \hat{E}_{a}\right)$ bases, where $\left.\hat{E}_{a}\right\lrcorner \hat{e}^{b}=\delta_{a}^{b}$. From Eqs. (1.5), (3.2), and (5.10), it follows that

$$
e^{a} \quad \text { and } \quad e^{4}:=\frac{\mathrm{d} r}{r}=\mathrm{d} \tau \quad \text { with } \quad \tau=\log r
$$

are invariant under the action of the finite group $\Gamma \subset$ $\mathrm{SU}(2)$.

\section{B. Nahm equations}

Consider the complex $\mathrm{V}$ bundle $\mathcal{E}$ over $\mathbb{C}^{2} / \Gamma$ described in Sec. III. Let

$$
\begin{aligned}
\mathcal{A}= & \hat{X}_{\mu} \hat{e}^{\mu} \\
= & \frac{1}{2}\left(\hat{X}_{1}-\mathrm{i} \hat{X}_{2}\right)\left(\hat{e}^{1}+\mathrm{i} \hat{e}^{2}\right)+\frac{1}{2}\left(\hat{X}_{3}-\mathrm{i} \hat{X}_{4}\right) \\
& \times\left(\hat{e}^{3}+\mathrm{i} \hat{e}^{4}\right)+\text { H.c. }
\end{aligned}
$$

be a connection on $\mathcal{E}$ written in the basis (5.8). The corresponding $\Gamma$-equivariance conditions are

$$
\begin{aligned}
& \gamma_{\Gamma}\left(\hat{X}_{1}-\mathrm{i} \hat{X}_{2}\right) \gamma_{\Gamma}^{-1}=\hat{X}_{1}-\mathrm{i} \hat{X}_{2} \quad \text { and } \\
& \gamma_{\Gamma}\left(\hat{X}_{3}-\mathrm{i} \hat{X}_{4}\right) \gamma_{\Gamma}^{-1}=\hat{X}_{3}-\mathrm{i} \hat{X}_{4} .
\end{aligned}
$$

Solutions to these equations are given by

$\frac{1}{2}\left(\hat{X}_{1}-\mathrm{i} \hat{X}_{2}\right)=\operatorname{diag}\left(\chi_{0}, \chi_{1}, \ldots, \chi_{k}\right)$ and

$\frac{1}{2}\left(\hat{X}_{3}-\mathrm{i} \hat{X}_{4}\right)=\operatorname{diag}\left(\varphi_{0}, \varphi_{1}, \ldots, \varphi_{k}\right)$,

$\frac{1}{2}\left(\hat{X}_{1}+\mathrm{i} \hat{X}_{2}\right)=-\operatorname{diag}\left(\chi_{0}^{\dagger}, \chi_{1}^{\dagger}, \ldots, \chi_{k}^{\dagger}\right)$ and

$\frac{1}{2}\left(\hat{X}_{3}+\mathrm{i} \hat{X}_{4}\right)=-\operatorname{diag}\left(\varphi_{0}^{\dagger}, \varphi_{1}^{\dagger}, \ldots, \varphi_{k}^{\dagger}\right)$,

where $\chi_{\ell}$ and $\varphi_{\ell}$ are $N_{\ell} \times N_{\ell}$ complex matrices. Thus, the $\Gamma$-equivariance conditions in the basis (5.8) force the block-diagonal form [Eq. (5.14)] of the connection components $\hat{X}_{\mu}$; i.e., the connection $\mathcal{A}$ is reducible, or else $N_{\ell}=0$ for $\ell \neq 0$, if $\Gamma$ acts trivially on $\mathcal{E}$.

The instanton equations (2.7) are conformally invariant, and it is more convenient to consider them on the cylinder

$$
\mathbb{R} \times S^{3} / \Gamma
$$

with the metric

$g_{\mathrm{cyl}}=\mathrm{d} \tau^{2}+\delta_{a b} e^{a} \otimes e^{b}=\frac{\mathrm{d} r^{2}}{r^{2}}+\delta_{a b} e^{a} \otimes e^{b}=\frac{1}{r^{2}} g$.

In the basis $\left(e^{\mu}\right)=\left(e^{a}, \mathrm{~d} \tau\right)$, the $\mathrm{SU}(2)$-invariant (spherically symmetric) connection $\mathcal{A}$ and its curvature $\mathcal{F}$ have components depending only on $r=\mathrm{e}^{\tau}$ and are given by

$$
\begin{aligned}
& \mathcal{A}=X_{\mu} e^{\mu} \quad \text { with } \quad X_{\mu}=r \hat{X}_{\mu}, \\
& \mathcal{F}_{4 a}=\frac{\mathrm{d} X_{a}}{\mathrm{~d} \tau}+\left[X_{\tau}, X_{a}\right] \quad \text { and } \\
& \mathcal{F}_{a b}=-2 \varepsilon_{a b c} X_{c}+\left[X_{a}, X_{b}\right],
\end{aligned}
$$

and Eq. (2.7) reduces to a form of the generalized Nahm equations given by

$\frac{\mathrm{d} X_{a}}{\mathrm{~d} \tau}=-\left[X_{4}, X_{a}\right]-2 X_{a}+\frac{1}{2} \varepsilon_{a b c}\left[X_{b}, X_{c}\right]-\Xi_{a}$.

Introducing

$$
Y_{\mu}:=\mathrm{e}^{2 \tau} X_{\mu} \quad \text { and } \quad s=\mathrm{e}^{-2 \tau}=\frac{1}{r^{2}},
$$

we obtain the equations

$$
2 \frac{\mathrm{d} Y_{a}}{\mathrm{~d} s}=\left[Y_{4}, Y_{a}\right]-\frac{1}{2} \varepsilon_{a b c}\left[Y_{b}, Y_{c}\right]+\frac{1}{s^{2}} \Xi_{a} .
$$

For $\Xi_{a}=0$, these equations coincide with the Nahm equations [25]. Choosing $\Xi_{a}=0$ and defining

$$
\alpha:=\frac{1}{2}\left(Y_{3}+\mathrm{i} Y_{4}\right) \quad \text { and } \quad \beta:=\frac{1}{2}\left(Y_{1}+\mathrm{i} Y_{2}\right),
$$

we obtain the equations

$$
\begin{gathered}
\frac{\mathrm{d}}{\mathrm{d} s}\left(\alpha+\alpha^{\dagger}\right)+\left[\alpha, \alpha^{\dagger}\right]+\left[\beta, \beta^{\dagger}\right]=0, \\
\frac{\mathrm{d} \beta}{\mathrm{d} s}+[\alpha, \beta]=0
\end{gathered}
$$

considered by Kronheimer [14,15] (see also Ref. [16]) in the description of SU(2)-invariant instantons. The equations (5.21) have three obvious solutions, which we now consider in turn.

\section{Abelian instantons with $\Xi_{a} \neq \mathbf{0}$}

For the first solution, we choose

$$
Y_{a}=-\frac{1}{2 s} \Xi_{a}=-\frac{r^{2}}{2} \Xi_{a} \text { and } Y_{4}=0 .
$$

Then we get the solution

$$
\mathcal{A}=-\frac{1}{2} r^{2} e^{a} \Xi_{a} \quad \text { and } \quad \mathcal{F}=\frac{1}{2} \eta_{\mu \nu}^{a} \Xi_{a} \hat{e}^{\mu} \wedge \hat{e}^{\nu}
$$

of the equations (2.7), which coincide with Eqs. (4.19) and (4.20). This configuration can also be regarded as a translationally equivariant solution of the self-dual Yang-Mills equations

$$
* \mathcal{F}=\mathcal{F}
$$

i.e., as an anti-instanton on $\mathbb{R}^{4} / \Gamma$ or $T^{4} / \Gamma$. 


\section{Abelian instantons with poles}

For the second solution, considered in Ref. [15], we set $\Xi_{a}=0$ and $\frac{\mathrm{d} Y_{a}}{\mathrm{~d} s}=0$. Then the constant matrices $Y_{\mu}$ satisfy the reduced anti-self-dual Yang-Mills equations

$$
\left[Y_{a}, Y_{4}\right]+\frac{1}{2} \varepsilon_{a b c}\left[Y_{b}, Y_{c}\right]=0
$$

considered in Ref. [10] and discussed in Sec. IV. Solutions to Eq. (5.28) are necessarily given by commuting matrices [10], and one can choose them in the forms

$$
Y_{a}=2 \Lambda^{2} \hat{\Xi}_{a} \text { and } Y_{4}=0,
$$

where $\hat{\Xi}_{a}$ have the form (3.10) and $\Lambda$ is a scale parameter. For the corresponding gauge potential and its field strength, we obtain

$$
\begin{gathered}
\mathcal{A}=X_{a} e^{a}=\frac{2 \Lambda^{2}}{r^{3}} \hat{\Xi}_{a} \hat{e}^{a} \quad \text { and } \\
\mathcal{F}=-\frac{2 \Lambda^{2}}{r^{4}} \bar{\eta}_{\mu \nu}^{a} \hat{\Xi}_{a} \hat{e}^{\mu} \wedge \hat{e}^{\nu},
\end{gathered}
$$

where $\hat{e}^{\mu}$ are given in Eq. (5.8) and $\bar{\eta}_{\mu \nu}^{a}$ are the anti-selfdual 't Hooft tensors defined by

$$
\bar{\eta}_{b c}^{a}=\varepsilon_{b c}^{a} \quad \text { and } \quad \bar{\eta}_{b 4}^{a}=-\bar{\eta}_{4 b}^{a}=-\delta_{b}^{a} .
$$

Thus, we obtain singular Abelian solutions with deltafunction sources in the Maxwell equations, as discussed by Ref. [15]. The gauge potential $\mathcal{A}$ from Eq. (5.30) can be regarded as an asymptotic approximation of a smooth solution. Note also that

$$
\bar{\omega}^{a}:=-\frac{2 \Lambda^{2}}{r^{4}} \bar{\eta}_{\mu \nu}^{a} \hat{e}^{\mu} \wedge \hat{e}^{\nu}
$$

can be viewed as three additional anti-self-dual symplectic forms on the cone $\left(\mathbb{R}^{4} \backslash\{0\}\right) / \Gamma=C\left(S^{3} / \Gamma\right)$, complementary to those given in Eq. (2.1).

\section{E.' 't Hooft instantons on $\mathbb{C}^{2} / \Gamma$}

For the third solution, we choose $Y_{4}=Y_{\tau}=0=\Xi_{a}$ to get

$$
\begin{aligned}
Y_{a} & =\frac{2}{s+\Lambda^{-2}} I_{a}=\frac{2 \Lambda^{2} r^{2}}{r^{2}+\Lambda^{2}} I_{a} \quad \text { with } \quad \Lambda \in \mathbb{R} \quad \text { and } \\
{\left[I_{a}, I_{b}\right] } & =\varepsilon_{a b}^{c} I_{c} .
\end{aligned}
$$

Then, for the anti-self-dual connection and curvature we obtain

$$
\begin{aligned}
& \mathcal{A}=\frac{2 \Lambda^{2}}{r^{2}+\Lambda^{2}} e^{a} I_{a} \quad \text { and } \\
& \mathcal{F}=-\frac{2 \Lambda^{2}}{\left(r^{2}+\Lambda^{2}\right)^{2}} \bar{\eta}_{\mu \nu}^{a} I_{a} \hat{e}^{\mu} \wedge \hat{e}^{\nu},
\end{aligned}
$$

where we used the relation $s=r^{-2}$. Here, $I_{a}$ are the generators of the group $\mathrm{SU}(2)$ embedded in the broken gauge group (3.11); i.e., there are $k+1$ instanton solutions with gauge group $\mathrm{SU}(2) \subset \mathrm{U}\left(N_{\ell}\right)$ if $N_{\ell} \geq 2$ for all $\ell=$ $0,1, \ldots, k$. From the explicit form of $e^{a}$ in Eq. (5.3), it follows that each of these solutions is the standard ' $t$ Hooft instanton generalized from $\mathbb{R}^{4}$ to $\mathbb{R}^{4} / \Gamma$. For framed instantons, ${ }^{7}$ there are four moduli: the scale parameter $\Lambda$ and three global $\mathrm{SU}(2)$ rotational parameters (see e.g., Ref. [22]).

\section{F. Moduli spaces of SU(2)-invariant instantons}

In the special case where $\Gamma$ is the trivial group, we obtain SU(2)-invariant solutions of the anti-self-dual Yang-Mills equations (4.18) on $\mathbb{R}^{4} \backslash\{0\}=C\left(S^{3}\right)$. The moduli spaces of these framed instantons (subject to appropriate boundary conditions) are four-dimensional hyper-Kähler ALE spaces $M_{\xi}$ resolving $M_{0}=\mathbb{C}^{2} / \Gamma^{\prime}$ as in Eq. (4.12), where $\Gamma^{\prime}$ is a finite subgroup of the group $\mathrm{SU}(2)$ related to boundary conditions for the solutions [14-16]. This is the moduli space of the spherically symmetric instanton which has the minimal topological charge $c_{2}(\mathcal{E})=\left(\# \Gamma^{\prime}-\right.$ $1) / \# \Gamma^{\prime}$. In our reducible case, we obtain a product of hyper-Kähler moduli spaces

$$
M_{\xi_{0}} \times M_{\xi_{1}} \times \cdots \times M_{\xi_{k}} .
$$

Note that $M_{\xi_{\ell}}$ is a point if $N_{\ell}=1$. For $N_{\ell}=1$, one can also use the singular Abelian solution from Eq. (5.30),

$$
\mathcal{F}^{\ell}=-\frac{2 \Lambda^{2}}{r^{4}} \bar{\eta}_{\mu \nu}^{a} \xi_{a}^{\ell} \hat{e}^{\mu} \wedge \hat{e}^{\nu},
$$

with $\xi_{a}^{\ell} \in \mathbb{R}$.

We have seen that for constant matrices $Y_{a}, Y_{\tau}$, the moduli space is the orbifold $M_{0}=\mathbb{C}^{2} / \Gamma$. For $s$-dependent solutions $Y_{a}, Y_{\tau}$, similarly to Refs. [10,16], one can choose boundary conditions such that each block tends to a constant multiple of the identity $\mathbf{1}_{N_{\ell}}$ in the limits $\tau \rightarrow \pm \infty$, while as $\tau \rightarrow 0$ the solutions define a representation of $\mathrm{SU}(2)$. For $N_{0}=N_{1}=\cdots=N_{k}=1$, it is natural to expect that the corresponding moduli space of solutions is a resolution of the orbifold $\mathbb{C}^{2} / \Gamma$.

\section{G. BPST instantons on $\mathbb{C}^{2} / \Gamma$}

Instead of the one-forms [Eq. (5.3)], one can introduce a basis of right $\mathrm{SU}(2)$-invariant one-forms on $S^{3} / \Gamma$ given by

$$
\tilde{e}^{a}:=-\frac{1}{r^{2}} \bar{\eta}_{\mu \nu}^{a} y^{\mu} \mathrm{d} y^{\nu} .
$$

They are dual to the vector fields $\tilde{E}^{a}$ given in Eq. (1.3), and they satisfy the relations

$$
\mathrm{d} \tilde{e}^{a}+\varepsilon_{b c}^{a} \tilde{e}^{b} \wedge \tilde{e}^{c}=0,
$$

\footnotetext{
${ }^{7}$ Framed instantons are instanton solutions modulo SU(2)invariant gauge transformations which approach the identity at asymptotic infinity.
} 


$$
g=\delta_{\mu \nu} \mathrm{d} y^{\mu} \otimes \mathrm{d} y^{\nu}=\mathrm{d} r^{2}+r^{2} \delta_{a b} \tilde{e}^{a} \otimes \tilde{e}^{b},
$$

which are similar to those for $e^{a}$ and can be proven by using identities for $\bar{\eta}_{\mu \nu}^{a}$ analogous to Eqs. (5.4) and (5.5).

The complex combinations

$$
\begin{aligned}
& \tilde{e}^{1}+\mathrm{i} \tilde{e}^{2}=\frac{\mathrm{i}}{r^{2}}\left(z^{1} \mathrm{~d} \bar{z}^{\overline{2}}-\bar{z}^{\overline{2}} \mathrm{~d} z^{1}\right) \quad \text { and } \\
& \tilde{e}^{3}+\mathrm{i} \tilde{e}^{4}=\frac{\mathrm{i}}{r^{2}}\left(\bar{z}^{\overline{1}} \mathrm{~d} z^{1}+z^{2} \mathrm{~d} \bar{z}^{\overline{2}}\right)
\end{aligned}
$$

are neither $(1,0)$ - nor $(0,1)$-forms with respect to the complex structure $J=J^{3}$. One can show that the forms in Eq. (5.40) are (1,0)-forms with respect to the complex structure

$$
\tilde{J}=\tilde{J}^{3}:=\left(\bar{\eta}_{\mu \lambda}^{3} \delta^{\lambda \nu}\right),
$$

which is used in the consideration of self-duality equations (and anti-instantons) on $\mathbb{R}^{4} / \Gamma$. The one-forms in Eqs. (5.10) and (5.40) are related by the coordinate change $z^{2} \mapsto \bar{z}^{\overline{2}}$ or, equivalently, by the change of orientation $x^{4} \mapsto-x^{4}$ of $\mathbb{R}^{4} / \Gamma$. For a fixed orientation, this inequivalence becomes more apparent in the case of the $\mathbb{C} P^{2}, K 3$, and ALE hyper-Kähler manifolds. Note that exactly $\tilde{e}^{a}$ (but not $e^{a}$ ) form a basis of one-forms on the SasakiEinstein space $S^{3} / \Gamma \subset \mathbb{R}^{4} / \Gamma$, since the complex structure on $\mathbb{C} P^{1} \hookrightarrow S^{3} / \Gamma$ is matched with Eqs. (5.39), (5.40), and (5.41) but not with Eqs. (2.5) or (5.10). In any case, $\tilde{e}^{a}$ are suitable one-forms on $\mathbb{R}^{4} / \Gamma$ which can be used in the ansatz for instanton solutions.

Let

$$
\mathcal{A}=\tilde{X}_{\mu} \tilde{e}^{\mu}
$$

be an $\mathrm{SU}(2)$-invariant connection on the $\mathrm{V}$ bundle $\mathcal{E}$ over $\mathbb{R}^{4} / \Gamma$ given in Eq. (3.7). Here $\tilde{e}^{a}$ are given in Eq. (5.37), $\tilde{e}^{4}:=\mathrm{d} \tau=\mathrm{d} r / r$ and $\tilde{X}_{\mu}$ depend only on $r=\mathrm{e}^{\tau}$. The explicit form [Eq. (5.40)] of $\tilde{e}^{\mu}$ and the $\Gamma$ action [Eq. (3.2)] imply $\Gamma$-equivariance conditions for the components $\tilde{X}_{\mu}$ given by

$$
\begin{aligned}
& \gamma_{\Gamma}\left(\tilde{X}_{1}+\mathrm{i} \tilde{X}_{2}\right) \gamma_{\Gamma}^{-1}=\zeta^{-2}\left(\tilde{X}_{1}+\mathrm{i} \tilde{X}_{2}\right) \quad \text { and } \\
& \gamma_{\Gamma}\left(\tilde{X}_{3}+\mathrm{i} \tilde{X}_{4}\right) \gamma_{\Gamma}^{-1}=\tilde{X}_{3}+\mathrm{i} \tilde{X}_{4} .
\end{aligned}
$$

For $k \geq 2$, the nonzero blocks of $\tilde{X}_{\mu}$ solving Eq. (5.43) are given by the matrix elements

$$
\begin{aligned}
\left(\tilde{X}_{1}+\mathrm{i} \tilde{X}_{2}\right)^{\ell, \ell+2} & \in \operatorname{Hom}\left(\mathbb{C}^{N_{\ell+2}}, \mathbb{C}^{N_{\ell}}\right) \text { and } \\
\left(\tilde{X}_{3}+\mathrm{i} \tilde{X}_{4}\right)^{\ell, \ell} & \in \operatorname{End}\left(\mathbb{C}^{N_{\ell}}\right)
\end{aligned}
$$

for $\ell=0,1, \ldots, k$, together with corresponding nonzero blocks of $\tilde{X}_{1}-\mathrm{i} \tilde{X}_{2}=-\left(\tilde{X}_{1}+\mathrm{i} \tilde{X}_{2}\right)^{\dagger}$ and $\tilde{X}_{3}-\mathrm{i} \tilde{X}_{4}=$ $-\left(\tilde{X}_{3}+\mathrm{i} \tilde{X}_{4}\right)^{\dagger}$.

In the following, we consider only the case of even rank $k=2 q$, since the odd case $k=2 q+1$ can be reduced to a "doubling" of the even case. Using the property $\xi^{2 q+1}=1$, one has $\operatorname{diag}\left(1, \zeta^{2}, \ldots, \zeta^{2 k}\right)=\operatorname{diag}\left(1, \zeta^{2}, \ldots, \zeta^{2 q}, \zeta, \zeta^{3}, \ldots, \zeta^{2 q-1}\right)$.

Then, by using the matrix

$$
\begin{gathered}
\gamma_{\Gamma}=\operatorname{diag}\left(\mathbf{1}_{N_{0}}, \zeta^{2} \mathbf{1}_{N_{1}}, \ldots, \zeta^{2 q} \mathbf{1}_{N_{q}}, \zeta \mathbf{1}_{N_{q+1}},\right. \\
\left.\zeta^{3} \mathbf{1}_{N_{q+2}}, \ldots, \zeta^{2 q-1} \mathbf{1}_{N_{2 q}}\right)
\end{gathered}
$$

in Eq. (5.43), we obtain the solution

$$
\begin{aligned}
& \tilde{X}_{1}+\mathrm{i} \tilde{X}_{2}=\left(\begin{array}{ccccc}
0 & \phi_{1} & 0 & \cdots & 0 \\
0 & 0 & \phi_{2} & \ddots & \vdots \\
\vdots & \vdots & \ddots & \ddots & 0 \\
0 & 0 & \cdots & 0 & \phi_{k} \\
\phi_{k+1} & 0 & \cdots & 0 & 0
\end{array}\right) \text { and } \\
& \tilde{X}_{3}+\mathrm{i} \tilde{X}_{4}=\left(\begin{array}{cccc}
\rho_{0} & 0 & \cdots & 0 \\
0 & \rho_{1} & \ddots & 0 \\
\vdots & \ddots & \ddots & 0 \\
0 & \cdots & 0 & \rho_{k}
\end{array}\right)
\end{aligned}
$$

where $\phi_{\ell+1} \in \operatorname{Hom}\left(\mathbb{C}^{N_{\ell+1}}, \mathbb{C}^{N_{\ell}}\right)$ and $\rho_{\ell} \in \operatorname{End}\left(\mathbb{C}^{N_{\ell}}\right)$.

In the basis $\left(\tilde{e}^{\mu}\right)=\left(\tilde{e}^{a}, \mathrm{~d} \tau\right)$, the $\mathrm{SU}(2)$-invariant connection $\mathcal{A}$ and the curvature $\mathcal{F}$ are given by

$$
\begin{gathered}
\mathcal{A}=\tilde{X}_{\mu} \tilde{e}^{\mu}=\hat{\tilde{X}}_{\mu} \hat{\tilde{e}}^{\mu} \text { with } \\
\hat{\tilde{X}}_{\mu}=\frac{1}{r} \tilde{X}_{\mu} \text { and } \\
\hat{\tilde{e}}^{\mu}=r \tilde{e}^{\mu}, \\
\mathcal{F}=\frac{1}{r^{2}}\left(\frac{1}{4} \varepsilon_{a b c}\left[\tilde{X}_{b}, \tilde{X}_{c}\right]-\tilde{X}_{a}\right) \bar{\eta}_{\mu \nu}^{a} \mathrm{~d} y^{\mu} \wedge \mathrm{d} y^{\nu} \\
+\left(\frac{\mathrm{d} \tilde{X}_{a}}{\mathrm{~d} \tau}+\left[\tilde{X}_{4}, \tilde{X}_{a}\right]-2 \tilde{X}_{a}+\frac{1}{2} \varepsilon_{a b c}\left[\tilde{X}_{b}, \tilde{X}_{c}\right]\right) \tilde{e}^{4} \wedge \tilde{e}^{a},
\end{gathered}
$$

where we used the identity

$$
\eta_{\mu \nu}^{a} \tilde{e}^{\mu} \wedge \tilde{e}^{\nu}=\frac{1}{r^{2}} \bar{\eta}_{\mu \nu}^{a} \mathrm{~d} y^{\mu} \wedge \mathrm{d} y^{\nu} .
$$

From Eq. (5.49), it follows that $\mathcal{F}$ is anti-self-dual, $* \mathcal{F}=-\mathcal{F}$, if $\tilde{X}_{a}$ satisfy the Nahm equations

$$
\frac{\mathrm{d} \tilde{X}_{a}}{\mathrm{~d} \tau}=2 \tilde{X}_{a}-\frac{1}{2} \varepsilon_{a b c}\left[\tilde{X}_{b}, \tilde{X}_{c}\right]-\left[\tilde{X}_{4}, \tilde{X}_{a}\right] .
$$

We obtain a solution by choosing $\tilde{X}_{4}=0$ and taking

$$
\tilde{X}_{a}=\frac{2 r^{2}}{r^{2}+\Lambda^{2}} I_{a} \quad \text { with } \quad\left[I_{a}, I_{b}\right]=\varepsilon_{a b}^{c} I_{c},
$$

where the $I_{a}$ are $\mathrm{SU}(2)$ generators in the irreducible representation on the space $\mathbb{C}^{N}$ with $N=N_{0}+N_{1}+\cdots+N_{k}$ 
that fits with the $\Gamma$-equivariant form [Eq. (5.47)]. For instance, one can work in the Coulomb branch with $N_{\ell}=1$ for all $\ell=0,1, \ldots, k$ so that $I_{a}$ embed the group SU(2) into $\mathrm{SU}(k+1)$. We thus obtain the configuration

$$
\begin{aligned}
& \mathcal{A}=-\frac{2}{r^{2}+\Lambda^{2}} \bar{\eta}_{\mu \nu}^{a} I_{a} y^{\mu} \mathrm{d} y^{\nu} \quad \text { and } \\
& \mathcal{F}=-\frac{2 \Lambda^{2}}{\left(r^{2}+\Lambda^{2}\right)^{2}} \bar{\eta}_{\mu \nu}^{a} I_{a} \mathrm{~d} y^{\mu} \wedge \mathrm{d} y^{\nu},
\end{aligned}
$$

which is exactly the BPST instanton extended from $\mathbb{R}^{4}$ to $\mathbb{R}^{4} / \Gamma$. We again have four moduli: the scale parameter $\Lambda$ and the three parameters of global SU(2) rotations.

The 't Hooft instanton [Eq. (5.34)] is gauge equivalent to the BPST instanton [Eq. (5.53)] on the Euclidean space $\mathbb{R}^{4}$. However, this is not so on the orbifold $\mathbb{R}^{4} / \Gamma$. For instance, taking $N_{\ell}=1$ for $\ell=0,1, \ldots, k$, one can obtain only Abelian solutions in the 't Hooft ansatz [Eq. (5.17)] while one has irreducible non-Abelian BPST instantons [Eq. (5.53)]. Of course, one can transform the solution in
Eq. (5.53) to a 't Hooft-type solution in a singular gauge, but this transformed solution will not be compatible with $\Gamma$-equivariance; i.e., it cannot be projected from $\mathbb{R}^{4}$ to $\mathbb{R}^{4} / \Gamma$. On the other hand, 't Hooft-type solutions are well defined on $\mathrm{V}$ bundles $\mathcal{E}$ over the orbifold $\mathbb{R}^{4} / \Gamma$ if the group $\Gamma$ acts trivially on the fibers of $\mathcal{E}$; i.e., if $\mathcal{E}=\mathcal{E}_{0}, N=N_{0}$, and $\gamma_{\Gamma}=\mathbf{1}_{N_{0}}$. The explicit form of such solutions for $N=N_{0}=2$ can be found, e.g., in Refs. [22,26,27].

\section{ACKNOWLEDGMENTS}

The work of T. A. I. and O. L. was partially supported by the Heisenberg-Landau program. The work of O.L. and A.D.P. was supported in part by the Deutsche Forschungsgemeinschaft under Grant No. LE 838/13. The work of R.J.S. was partially supported by Consolidated Grant No. ST/J000310/1 from the U.K. Science and Technology Facilities Council, and by Grant No. RPG-404 from the Leverhulme Trust.
[1] A. A. Belavin, A. M. Polyakov, A. S. Schwarz, and Y.S. Tyupkin, Phys. Lett. 59B, 85 (1975).

[2] T. Eguchi and A. J. Hanson, Phys. Lett. 74B, 249 (1978); Gen. Relativ. Gravit. 11, 315 (1979).

[3] G. W. Gibbons and S. W. Hawking, Phys. Lett. 78B, 430 (1978).

[4] T. Eguchi, P. B. Gilkey, and A. J. Hanson, Phys. Rep. 66, 213 (1980).

[5] N. Manton and P. Sutcliffe, Topological Solitons (Cambridge University Press, Cambridge, 2004).

[6] E.J. Weinberg, Classical Solutions in Quantum Field Theory (Cambridge University Press, Cambridge, 2012).

[7] R. S. Ward, Phys. Lett. 61A, 81 (1977).

[8] M. F. Atiyah, N. J. Hitchin, and I. M. Singer, Proc. R. Soc. A 362, 425 (1978).

[9] M.F. Atiyah, N. J. Hitchin, V. G. Drinfeld and Yu. I. Manin, Phys. Lett. 65A, 185 (1978).

[10] P. B. Kronheimer, J. Diff. Geom. 29, 665 (1989).

[11] N. J. Hitchin, A. Karlhede, U. Lindstrom, and M. Rocek, Commun. Math. Phys. 108, 535 (1987).

[12] M. R. Douglas and G. W. Moore, arXiv:hep-th/9603167.

[13] N. J. Hitchin, Asterisque 206, 137 (1992).
[14] P. B. Kronheimer, J. Diff. Geom. 32, 473 (1990).

[15] P. B. Kronheimer, J. Lond. Math. Soc. s2-42, 193 (1990).

[16] R. Bielawski, J. Lond. Math. Soc. 55, 400 (1997); Ann. Glob. Anal. Geom. 14, 177 (1996).

[17] P. B. Kronheimer and H. Nakajima, Math. Ann. 288, 263 (1990).

[18] M. K. Prasad, Physica (Amsterdam) D 1, 167 (1980).

[19] M. R. Douglas and N. A. Nekrasov, Rev. Mod. Phys. 73, 977 (2001).

[20] R. J. Szabo, Phys. Rep. 378, 207 (2003).

[21] H. Nakajima, Duke Math. J. 76, 365 (1994).

[22] M. Bianchi, F. Fucito, G. Rossi, and M. Martellini, Nucl. Phys. B473, 367 (1996).

[23] S. K. Donaldson, Proc. London Math. Soc. s3-50, 1 (1985).

[24] K. Uhlenbeck and S.-T. Yau, Commun. Pure Appl. Math. 39, S257 (1986).

[25] W. Nahm, Report No. CERN-TH-3172 (to be published); Lect. Notes Phys. 180, 456 (1983).

[26] D. M. Austin, J. Diff. Geom. 32, 383 (1990).

[27] G. Etesi and T. Hausel, Commun. Math. Phys. 235, 275 (2003); G. Etesi, Nucl. Phys. B662, 511 (2003). 